\title{
Does quality of developmental care in NICUs affect health-related quality of life in 5-y-old children born preterm?
}

\author{
Rosario Montirosso', Lorenzo Giusti' , Alberto Del Prete ${ }^{2}$, Rinaldo Zanini ${ }^{2}$, Roberto Bellù ${ }^{2}$ and Renato Borgatti ${ }^{3}$; \\ NEO-ACQUA Study Group
}

BACKGROUND: Preterm birth and Neonatal Intensive Care Unit (NICU) stay are early adverse experiences, which may affect health-related quality of life (HRQoL) even in the absence of prematurity-related morbidities. The aim of this multicenter longitudinal study was to examine the relation between quality levels of NICU Developmental Care (DC) and HRQoL at 60 mo in children who were born preterm.

METHODS: HRQOL of 102 very preterm (VPT) children from 20 NICUs and 110 full-term controls was assessed using TNO-AZL Preschool Children's Quality of Life Questionnaire (TAPQOL). In VPT children, we compared HRQoL by splitting NICUs into units with high- and low-quality of DC according to the following two factors: (i) the infant centered care (ICC), and (ii) the infant pain management (IPM).

RESULTS: Compared to VPT children from NICUs with highquality of ICC, VPT children from NICUs with low-quality in ICC scored lower in HRQoL component which resulted from the aggregation of lively, positive emotionality, social and motor functioning. No differences were found between VPT children from high-quality ICC NICUs and full-term children and for the IPM index.

CONCLUSION: Findings suggest that higher quality of DC in $\mathrm{NICU}$ related to ICC might mitigate long-term negative quality of life outcomes.

V ery preterm infants (VPT) are at high risk for developing long-term neurodevelopmental impairment, including low health-related quality of life (HRQoL, ref. (1)). Compared with controls, preschool-aged children who were born preterm exhibited poorer performance in motor (2), social, (3) and emotional functioning (4). Importantly, while HRQoL outcomes are related to severe clinical complications in perinatal period (5), lower scores are also reported in children without morbidities, suggesting that HRQoL could also be affected by other factors than severity of prematurity-related morbidities (6). As early experience has profound effect on the developing brain, Developmental Care (DC) interventions provided in NICU may support neurodevelopment which in turn might improve longterm HRQoL. Studies suggest that DC interventions improve infant's neurodevelopment on the short- (7) and long-term (8). DC includes a broad category of interventions designed to minimize the effects of NICU stress exposure (9), such as: control of external stimuli, kangaroo care and parental involvement, developmental activities of daily living, and infant pain management (10). A critical point is that the utilization of DC practices is not yet uniformly applied in NICUs and multicenter research offers unique insight about the variability between units (11). Broad variations among units have been documented in parental visiting policies (12), in breastfeeding support (13) and in the use of mechanical lance and nonpharmacological analgesia (14). Consequently, it is possible that NICUs integrate only some practices of DC into their conventional care, resulting in different levels of DC quality provided. Differences in the actual level of DC incorporated in a NICU's standard care have been evaluated in relation to VPT infants' neurobehavioral profile during the neonatal period (15) and in toddlerhood (16). However, it is unknown whether DC levels routinely used in NICUs contribute to lasting effects on HRQoL during preschool age.

We evaluated the relationships between the quality of DC carried out in 20 Italian NICUs and HRQoL at 60 mo of age in children born VPT, including an age-matched sample of fullterm children as control. To evaluate the level of DC in NICUs, we used a specifically developed questionnaire, the Neonatal Adequate Care for Quality of Life (NEO-ACQUA) Quality of Care Questionnaire (QCQ). We compared HRQoL of children by splitting the NICUs into units with high and low quality of DC and then examining if scores obtained in the HRQoL scales were associated with levels of quality of care. We hypothesized that children from NICUs with high-quality of DC had a better HRQoL outcome than children from NICUs with lowquality of DC. Furthermore, we expected that children from NICUs characterized by high-quality of DC would exhibit scores similar to those displayed by a control group of fullterm children. Previous research has documented that at-risk preschoolers have poorer HRQoL (17). Furthermore, parental stress has been associated with lower ratings of quality of life (18) and maternal distress may partly contribute to higher perceptions of HRQoL difficulties in preterm children (4). In order to assess the potential contribution of these variables, we

${ }^{1} 0-3$ Centre for the at-Risk Infant, Scientific Institute, IRCCS Eugenio Medea, Bosisio Parini, Lecco, Italy; ${ }^{2}$ Department of Neonatal Care and Neonatal Intensive Care Unit, Manzoni Hospital, Lecco, Italy; ${ }^{3}$ Neuropsychiatry and Neurorehabilitation Unit, Scientific Institute, IRCCS Eugenio Medea, Lecco, Italy. NEO-ACQUA Study Group is listed in the Appendix. Correspondence: Rosario Montirosso (rosario.montirosso@bp.Inf.it)

Received 22 April 2016; accepted 16 June 2016; advance online publication 31 August 2016. doi:10.1038/pr.2016.158 
preliminarily compared child development level and parental stress among the three groups.

\section{RESULTS}

Descriptive statistics are presented for each variable in Tables 1 and 2. No differences were found between children from lowand high-care, for the infant pain management (IPM) and ICC indexes, in the general characteristics of the NICUs (i.e., admission for year, days of recovery for year, number of beds) and perinatal data. There were no significant differences in age, education, family SES, and PSI-SF scores among the mothers of children from the low-care, high-quality care and control groups. No significant effects both for ICC and IPM index were found on General Development score among three groups.

Principal components analysis applied to TAPQOL scales yielded a four-factor solution (eigenvalues $>1.0$ ) representing $57.11 \%$ of the total variance. An examination of the scree plot revealed a steeper decline from the second to the third eigenvalue, suggesting that only two factors had to be rotated. The rotated matrix showed that the first factor, which accounted for $21.67 \%$ of the variance, included the following scales: stomach (0.67), sleep (0.62), appetite (0.70), behavior (0.69), and anxiety (0.49). It represents a physical-emotional component (PEC) of HRQoL, assessing child's negative emotions (e.g., frighten, tense), behavior problems (e.g., irritability), and some functional problems, such as quality of sleeping, eating difficulty, stomach problems (e.g., abdominal pain). The second factor, which accounted for $17.01 \%$ of the variance, included: lively (0.82), positive mood (0.73), social (0.70), and motor (0.58) scales. It represents a liveliness component (LC) of HRQoL, assessing child's liveliness (e.g., energetic, active), positive emotions (e.g., cheerful, happy), positive relationship with peers, and motor problems (e.g., difficulties in walking, running). No cross-loadings ( $>0.35$ ) were observed between

Table 1. Descriptive statistics of NICUs characteristics and perinatal variables subdivided for high and low quality of care, separately for infant centered care (ICC) index and infant pain management (IPM) index and for full-term children

\begin{tabular}{|c|c|c|c|c|c|c|c|c|c|c|}
\hline & \multicolumn{8}{|c|}{ Preterm group } & & \\
\hline & \multicolumn{4}{|c|}{ ICC index } & \multicolumn{4}{|c|}{ IPM index } & & \\
\hline & \multicolumn{2}{|c|}{$\begin{array}{c}\text { Low }(N I C U=10) \\
(N=57,31 \mathrm{~F})\end{array}$} & \multicolumn{2}{|c|}{$\begin{array}{l}\operatorname{High}(\mathrm{NICU}=10) \\
\quad(N=45,24 \mathrm{~F})\end{array}$} & \multicolumn{2}{|c|}{$\begin{array}{c}\text { Low }(\mathrm{NICU}=11) \\
(N=49,28 \mathrm{~F})\end{array}$} & \multicolumn{2}{|c|}{$\begin{array}{c}\text { High }(\mathrm{NICU}=9) \\
(N=53,27 \mathrm{~F})\end{array}$} & \multicolumn{2}{|c|}{$\begin{array}{l}\text { Full-term group } \\
(N=110,55 \mathrm{~F})\end{array}$} \\
\hline & Mean & SD & Mean & SD & Mean & SD & Mean & SD & Mean & SD \\
\hline \multicolumn{11}{|l|}{ NICUs variables } \\
\hline Number of beds & 20.33 & 10.04 & 20.64 & 12.49 & 19.73 & 9.47 & 8.00 & 13.49 & - & - \\
\hline \multicolumn{11}{|l|}{ Perinatal variables } \\
\hline Birth weight (g) & $1,163.88$ & 216.11 & $1,067.45$ & 247.50 & $1,122.88$ & 233.81 & $1,120.92$ & 236.65 & $3,290.09$ & 364.71 \\
\hline Gestational age at birth (weeks) & 29.12 & 1.96 & 28.73 & 2.34 & 29.06 & 2.08 & 28.85 & 2.20 & 39.34 & 1.17 \\
\hline
\end{tabular}

VON-RA, Vermont Oxford Network-Risk Adjustment index.

Table 2. Descriptive statistics of socio-demographic variables, parental stress and child development subdivided for high and low quality of care, separately for infant centered care (ICC) index and infant pain management (IPM) index and for full-term children

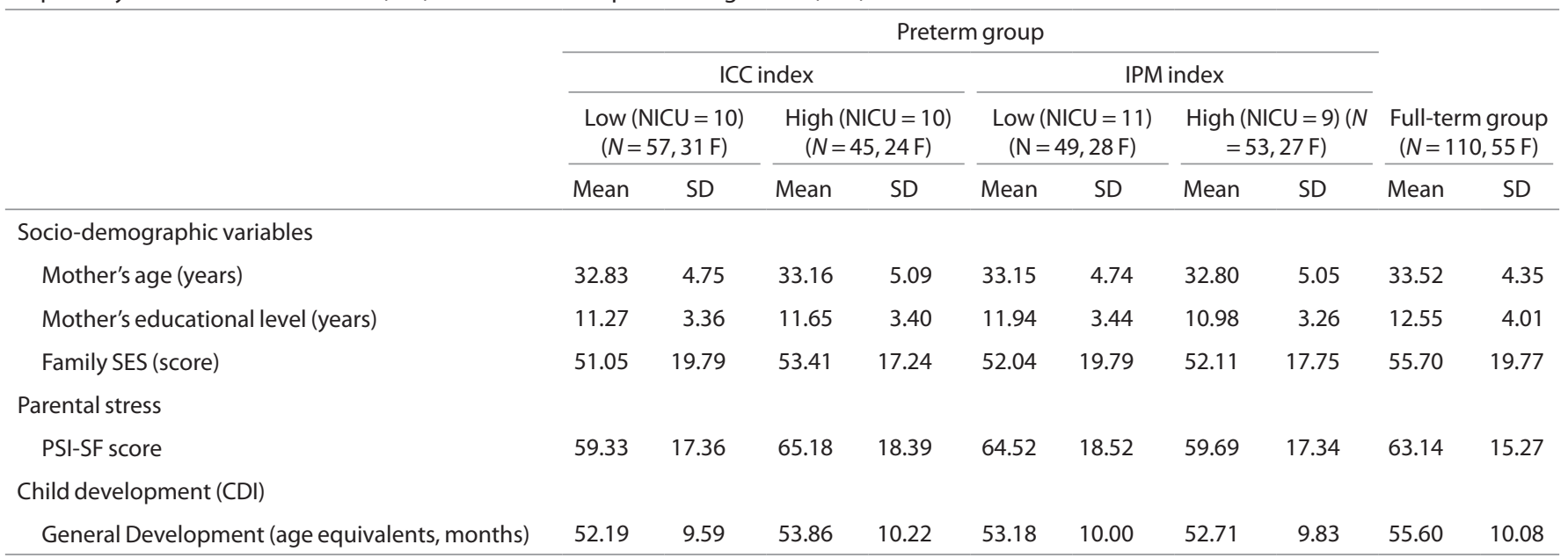

PSI-SF, Parental Stress Index-Short Form. 


\section{Articles | Montirosso et al.}

PEC and LC and, the internal consistency (Cronbach's alphas) were .65 and .60 , respectively. The correlation between the two factors was $r=-0.05, P>0.05$. Accordingly, two averaged scores were computed and used in further analyses.

A significant effect was observed for the ICC index on the LC factor, $F(2,211)=6.38, P=0.000, \eta_{\mathrm{p}}^{2}=0.050$. Post-hoc comparisons showed that preterm children from units with low-quality in ICC obtained lower scores than either children from ICC high-care units $(P=0.01)$ and control group children $(P=0.003)$. No significant differences emerged between children from ICC high-care units and full-terms both for PEC and LC (Figure 1). For the IPM index, no significant effects or interactions were found among the three groups for HRQoL components.

\section{DISCUSSION}

Children from NICUs with high-quality in parental involvement and nursing interventions aimed to promote infant stability scored higher in HRQoL LC factor compared to children from NICUs with low-quality in ICC. Like FT children, VPT children from ICC high-care units exhibited a good quality of life characterized by a general positivity, more vitality, more social functioning and, fewer difficulties in motor functioning. A previous study found that infants from NICUs with high-quality in ICC showed a better neurobehavioral profile at the discharge (15). Thus, DC procedures associated to ICC index promote neurodevelopment of these children, which in turn might support a better quality of life.

The finding that children from IPM high-care units reported similar HRQoL to children from IPM low-care units was unexpected. The result is not readily explained, given that previous research has provided evidence that high level of infant pain management in NICU was related to less internalizing problems at $18 \mathrm{mo}(16)$. A possible interpretation of the current finding is that potential positive effects of high-quality neonatal pain management might be transient, becoming less notable during preschool age.

The lack of significant findings for PEC needs additional comments. According to previous study (19), we found two different components of HRQoL which were not associated to each other. Thus, it should not be surprising that the ICC and IPM were not associated to similar HRQoL outcomes. Moreover, previous

a

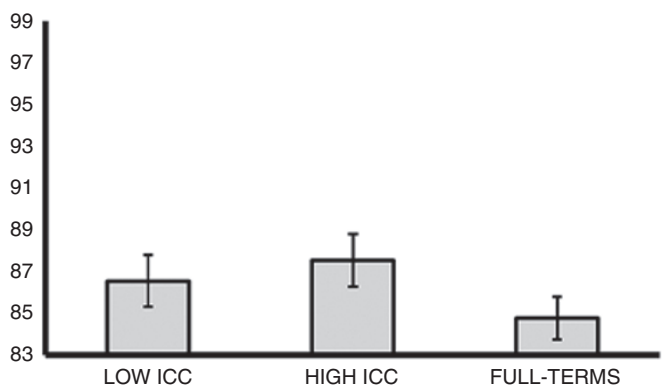

HRQoL studies investigating difference between VPT children and full-term controls at preschool age lead to contradictory findings on the domains covered by PEC, such as negative emotions, behavior problems, and some functional problems (1-4). It should be noted that the enrolled VPT children were free of major neurodevelopmental disabilities and TAPQOL does not assess only the presence of a problem, but give additional information about how children experience that problem. Indeed, our findings are at least partially consistent with previous studies using TAPQOL $(5,6,20)$, suggesting that in parents' perception both health status and related subjective feelings of their VPT children in physical-emotional component of HRQoL were comparable to those of children born full-term.

This study has limitations. First, the 20 included NICUs self-selected themselves into the NEO-ACQUA study. Thus, although the use of data from a wide number of NICUs represents a substantial strength of the study, the participating units cannot be considered representative of DC quality of about 130 Italian NICUs. Second, not all the aspects of DC were examined, and those that were not captured by two indexes (e.g., disruption of sleep) may have an impact on child outcomes. Third, the use of care-provider self-report tools to assess DC practices could attenuate the associations between the QCQ responses, actual clinical practice, and HRQoL outcomes. However, it is also important to take in consideration that: (i) care level at each NICU was established based on a statistical procedure (i.e., a median split), so the informants completing the QCQ were unaware of the care level assigned to units; (ii) QCQ data were collected separately from the HRQoL assessment, which was completed by parents unaware of all other information gathered via the QCQ. This methodological approach had the advantage of: (i) limiting bias in the NICUs sampling in term of high- and low-quality DC; (ii) reducing the risk of bias due to possible relationships between HRQoL assessment at $60 \mathrm{mo}$ of age and any clinical procedures in the participating NICUs.

Our findings suggest that DC procedures in NICU are associated with HRQoL outcomes beyond infancy. Importantly, general characteristics of the NICUs, perinatal variables, socio-demographic factors, parental stress and children development level did not differ between VPT children from NICUs with high- and lowquality of DC. Therefore, these factors did not appear sufficient

b

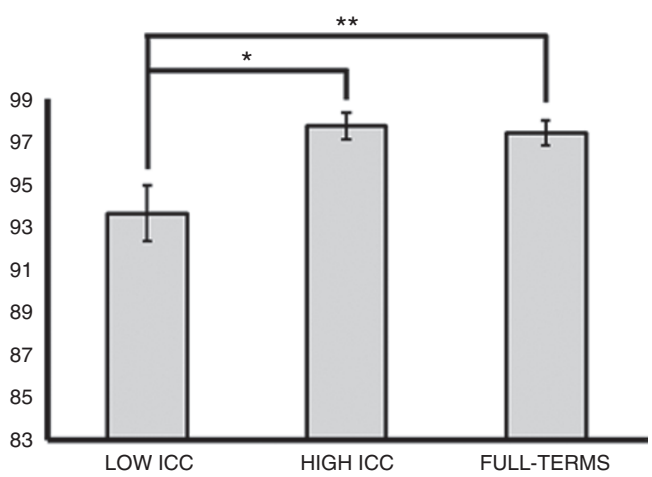

Figure 1. Health-related quality of life at 60 mo of age. Means of the scores for physical-emotional component (a) and liveliness component (b) of healthrelated quality of life in the preterm children from high- and low-care units for infant centered care index (ICC) and in full-term children. ${ }^{*} P=0.01$. ${ }^{*} P=0.003$. 
in-end-of themselves to account for the difference in HRQoL observed in children from NICUs with low-quality care. It is also worth noting that VPT children from NICUs with higher ICC obtained HRQoL scores similar to those reported for FT children. Thus, it seems important to encourage DC practices promoting parents' closeness and nursing procedures aimed to increase neurobehavioral stability of infants, which in turn might mitigate later HRQoL in VPT children. On the other hand, our results suggest that DC interventions routinely used in NICUs likely make differential contributions in preventing the development of later problematic child outcomes. Thus, it is important to highlight that likely not all of these practices have an equivalent impact or efficacy. In other words, a generic assumption that some DC procedures embedded in clinical practice are intrinsically helpful for child development would be, at the best, limited. More research is needed in this area to guide our understanding of which specific kind of DC routinely carried out in NICUs might effectively promote HRQoL in VPT children.

\section{METHODS}

\section{Participants}

The research is part of the NEO-ACQUA project, a multicenter, longitudinal study on the relationship between the quality of care received by infants in the NICU and their subsequent outcomes and quality of life (for details, see ref. (21)). The study design included behavioral, cognitive, and language follow-up assessments until $7 \mathrm{y}$ of age with VPT children compared with a control group of full-term (FT) peers. The current study is focused on data from the 60 mo follow-up HRQoL assessment. During the hospitalization, a group of 178 VPT infants were recruited consecutively from 25 Italian tertiary care NICUs between January 2006 and December 2007. Inclusion criteria for preterm infants were: gestational age between 22 and $29^{+6} \mathrm{wk}$ and/or birth weight between 401 and 1,500g. Exclusion criteria included: major brain lesions as documented by cerebral ultra-sound, neuro-sensorial deficits, genetic syndromes and/or major malformations. A group of $180 \mathrm{FT}$ infants were selected from the sequential register of births held in the delivery ward of the participating hospitals. For each VPT infant, the first suitable FT newborn born after each preterm infant, who was not admitted to the NICU, was enrolled. FT infants were healthy, with no pathologies and risk factors in pregnancy and during the perinatal period. FT infants were matched for gender, mothers' age, mothers' education, and family socio-economic status with VPT infants.

At the 60-mo-follow-up, five NICUs did not participate in the study for organizational reasons. From the original sample, 102 VPT infants and $110 \mathrm{FT}$ infants participated in the study, resulting in a 42.7 and a $38.9 \%$ attrition rate, respectively. Causes for lack of participation at the follow-up included children's illness and family-related reasons (i.e., impending relocation, lack of time for participation). No significant differences in gender, gestational age, birth weight, days of hospitalization, mothers' age, mothers' education, and family socioeconomic index were found between children participating and notparticipating in the follow-up session.

The study was approved by the Ethics Committees in each participating hospital. All parents signed a written informed consent form.

\section{Measures}

To evaluate the level of DC among NICUs, we used the NEO-ACQUA QCQ (21). The QCQ is a 29-item questionnaire designed to measure a variety of DC practices used in NICUs. For each NICU, a neonatologist with at least $5 \mathrm{y}$ of clinical experience, who was not involved in the direct care of the infants filled out the QCQ and rated the level of DC quality on items ranging from 0 (low quality of care) to 1 or 2 (high quality of care). In order to aggregate the items into a reduced number of DC descriptors, we applied factor analysis to QCQ responses, revealing a three-factor structure. The first factor, labeled nursing staffing, accounted for $12 \%$ of the variance, included items such as number of physicians per bed, number of graduate students, fellows, or consultants per bed, nurse chiefs per bed, nurses per bed, and nursery nurse, assistants per bed. Given the goal of this study, we used two indexes of DC: the infant centered care (ICC) index and the IPM index. The ICC accounted for $20 \%$ of the variance and included four items (Cronbach's $\alpha=0.84$ ): (1) parent's involvement in care, such as parents to spend the night in the unit; (ii) use of parental kangaroo care as a routine procedure; (iii) duration per day of kangaroo care ( $<$ or $\geq 45 \mathrm{~min}$ ); (iv) presence of nursing interventions to support infant development by decreasing infant energy expenditure and promoting stability (i.e., infant containment, postural maneuvers, and reduction of disturbing tactile stimulation). The IPM accounted for $9 \%$ of the variance and included five items (Cronbach's $\alpha=0.75$ ): (i) number of invasive medical procedures (e.g., intravenous lines, drainage tubes, endotracheal tubes, etc.) in which nonpharmacologic procedures (i.e., pacifier, glucose, containment with towels or blankets) are used for reducing pain; (ii) number of invasive medical procedures in which pharmacologic procedures are used for reducing pain; (iii) use of pharmacologic analgesia or sedation during continuous mechanical ventilation; (iv) use of a blood collection procedure (i.e., heel-stick by manual lance or mechanical one); (v) use of a clinical evaluation scale of infant pain and/or a protocol designed for pain management of newborn infants, including guidelines and well-established recommendations. Factor loadings were utilized to compute composite scores for ICC and IPM indexes for each NICU. The ICC index ranged from 0 to 8 , with higher scores indicating higher level of ICC. The IPM index ranged from 0 to 10 , with higher scores indicating higher levels of IPM.

Infants' perinatal variables including gestational age, birth weight and length of stay in the NICU were obtained from medical records. We also assessed infants' clinical status computing the Vermont Oxford Network Risk Adjustment (VON-RA; ref. (22)) index. Low VON-RA scores indicate less serious clinical outcomes.

Socio-demographic data such as maternal age, years of education, and occupational status were obtained for both parents through a questionnaire. According to Hollingshead's classification (23), the more prestigious occupational level between mother and father (i.e., the highest of the two ratings) was considered to indicate the family socio-economic status (SES). Score ranges from 0 (occupations that do not require high school graduation) to 90 (occupations that require highly specialized education and training). Lower scores reflected lower socio-economic status.

HRQoL was assessed using the TNO-AZL Preschool Children's Quality of Life Questionnaire for children aged 1-5 y (TAPQOL, ref. (2)). TAPQOL is a 43 -item questionnaire consisting of 12 multi-item scales: Sleeping, Appetite, Lungs, Stomach, Skin, Motor Functioning, Social Functioning, Problem Behavior, Communication, Anxiety, Positive Mood, Liveliness. Each scale is expressed in a score that varies from 0 to 100 with higher scores indicating better quality of life.

Child Development Inventory (CDI, ref. (24)) is a 270 -item parentreport assessment aimed at determining developmental outcomes in high-risk children aged 15 mo to 6 y (25). CDI measures eight domains of development: social, self-help, gross motor, fine motor, expressive language, language comprehension, letters, and numbers. CDI provides also an overall development index (i.e., General Development). For the aims of this study, we used the General Development scale with scores reported in terms of age equivalents (months).

Parenting Stress Index-Short Form (PSI-SF, ref. (26)) is a 36-item self-report questionnaire to measure the overall stress experienced in parenting. The PSI-SF provides a general score, which is calculated on the base of three subscales (i.e., Parental Distress, Parent-Child Dysfunctional Interaction, and Difficult Child). Higher PSI-SF score indicates higher parental stress.

\section{Data Analysis}

To examine differences within the VPT group, taking into consideration the level of DC quality, each NICU was assigned to a low- or high-quality of DC group, based on median splits for the ICC and IPM. For the ICC $($ median $=7.00$, mean $=6.86, S D=1.79)$, 10 NICUs had low-quality DC (60 infants), and 10 NICUs had high-quality DC (49 infants). For the IPM (median $=7.00$, mean $=6.08$, SD = 2.32), 11 NICUs had lowquality DC (51 infants) and 9 NICUs had high-quality DC (58 infants). Such dichotomization resulted in a categorical measure of high/low exposure to level of DC quality, avoiding bias from extreme scores. To 
determine the effects of quality of care (low-care group vs. high-care group vs. control group), categorical variables were analyzed by chisquare tests, while continuous variables were analyzed through separate ANOVAs with Bonferroni-corrected post-hoc tests, separately for IPM and ICC. A principal components analysis with Varimax rotation was conducted to aggregate TAPQOL scale scores using the control group data. Explained variance was computed for all factors with an eigenvalue greater than one. Additionally, we examined the plot of the eigenvalue (e.g., scree test). Factors loading $\geq 0.45$ were considered acceptable (see below). To determine the effects of DC quality of care (low-care group vs. high-care group vs. control group) on the HRQoL components, two one-way multivariate analyses of variance with Bonferroni-corrected post-hoc tests were conducted, separately for IPM and ICC.

\section{APPENDIX}

NEO-ACQUA Study Group:

Fabio Mosca, Odoardo Picciolini, NICU, Department of Maternal and Pediatric Sciences, University of Milan Fondazione IRCCS Ca' Granda, Milan, Italy; Stefano Visentin, Nadia Battajon, Neonatology and NICU, Ca'Foncello Hospital, Treviso, Italy;

Maria Lucia Di Nunzio, Fiorina Ramacciato, NICU, Cardarelli Hospital, Campobasso, Italy;

Laura Barberis, Emmanuele Mastretta, Division of Neonatology and NICU, S. Anna Hospital, Turin, Italy;

Giovanna Carli, Michela Alfiero Bordigato, NICU, Hospital of Camposampiero, Camposampiero Italy;

Valeria Chiandotto, Cristiana Boiti, Department of Neonatology, University Hospital S. M. M., Udine, Italy;

Rosangela Litta, Giovanna Minelli, Division of Neonatology and NICU, Ospedali Riuniti, Foggia, Italy;

Marcello Napolitano, NICU, Evangelic Hospital Villa Betania, Napoli, Italy;

Alessandro Arco, NICU, University Hospital G. Martino, Messina, Italy;

Palma Mammoliti, NICU, Ospedale degli Infermi, Rimini, Italy;

Cinzia Fortini, NICU, PediatricUniversity Hospital, Ferrara, Italy;

Paolo Tagliabue, Division of Neonatology, San Gerardo Hospital, Monza, Italy; Lorenzo Quartulli, NICU, Perrino Hospital, Brindisi, Italy;

Giuliana Motta, NICU, Niguarda Hospital Ca'Granda, Milan, Italy;

Paola Introvini, NICU, Buzzi Hospital, Milan, Italy;

Rosetta Grigorio, NICU, Umberto I Hospital, Siracusa, Italy;

Paola Mussini, NICU, C. Poma Hospital, Mantova, Italy;

Giulia Pomero, NICU, Santa Croce e Carle Hospital,Cuneo, Italy;

Carlo Poggiani, NICU, Istituti Ospitalieri, Cremona, Italy;

Ananda Bauchiero, Department of Neonatology, S. Anna University Hospital, Turin, Italy.

\section{ACKNOWLEDGMENTS}

The NEO-ACQUA project was supported by an advisory group whose membership consisted of the following people: Roberto Bellù, Renato Borgatti, Alberto Del Prete, Guido Calciolari, Maria Caterina Cavallo, Rosario Montirosso, and Rinaldo Zanini. Thanks go to the MediData Studi e Ricerche staff in Modena for their organizational, technical, and scientific support. We are very grateful to the staff of all NICUs. We would also like to thank participating children and their parents for their involvement in this study.

\section{STATEMENT OF FINANCIAL SUPPORT}

The NEO-ACQUA project was supported by an unrestricted educational grant from Chiesi Farmaceutici S.p.A., Parma, Italy.

Disclosure: The authors have stated that they had no interests which might be perceived as posing a conflict or bias.

\section{REFERENCES}

1. Zwicker JG, Harris SR. Quality of life of formerly preterm and very low birth weight infants from preschool age to adulthood: a systematic review. Pediatrics 2008;121:e366-76.

2. Fekkes M, Theunissen NC, Brugman E, et al. Development and psychometric evaluation of the TAPQOL: a health-related quality of life instrument for 1-5-year-old children. Qual Life Res 2000;9:961-72.

3. Klassen AF, Lee SK, Raina P, Chan HW, Matthew D, Brabyn D. Health status and health-related quality of life in a population-based sample of neonatal intensive care unit graduates. Pediatrics 2004;113(3 Pt 1):594-600.
4. Eiser C, Eiser JR, Mayhew AG, Gibson AT. Parenting the premature infant: balancing vulnerability and quality of life. J Child Psychol Psychiatry 2005;46:1169-77.

5. Huhtala M, Korja R, Rautava L, et al.; PIPARI Study Group. Health-related quality of life in very low birth weight children at nearly eight years of age. Acta Paediatr 2016;105:53-9.

6. Chien LY, Chou YH, Ko YL, Lee CF. Health-related quality of life among 3-4-year-old children born with very low birthweight. J Adv Nurs 2006;56:9-16.

7. Milgrom J, Newnham C, Anderson PJ, et al. Early sensitivity training for parents of preterm infants: impact on the developing brain. Pediatr Res 2010;67:330-5.

8. Spittle A, Orton J, Anderson P, Boyd R, Doyle LW. Early developmental intervention programmes post-hospital discharge to prevent motor and cognitive impairments in preterm infants. Cochrane Database Syst Rev 2012;12:CD005495.

9. Als H, Duffy FH, McAnulty GB, et al. Early experience alters brain function and structure. Pediatrics 2004;113:846-57.

10. Symington A, Pinelli JM. Distilling the evidence on developmental care: a systematic review. Adv Neonatal Care 2002;2:198-221.

11. Ashbaugh JB, Leick-Rude MK, Kilbride HW. Developmental care teams in the neonatal intensive care unit: survey on current status. J Perinatol 1999;19:48-52.

12. Greisen G, Mirante N, Haumont D, et al.; ESF Network. Parents, siblings and grandparents in the Neonatal Intensive Care Unit. A survey of policies in eight European countries. Acta Paediatr 2009;98:1744-50.

13. Bonet M, Marchand L, Kaminski M, et al.; "EDEN Mother-Child Cohort Study Group". Breastfeeding duration, social and occupational characteristics of mothers in the French 'EDEN mother-child' cohort. Matern Child Health J 2013;17:714-22.

14. Losacco V, Cuttini M, Greisen G, et al.; ESF Network. Heel blood sampling in European neonatal intensive care units: compliance with pain management guidelines. Arch Dis Child Fetal Neonatal Ed 2011;96:F65-8.

15. Montirosso R, Del Prete A, Bellù R, Tronick E, Borgatti R; Neonatal Adequate Care for Quality of Life (NEO-ACQUA) Study Group. Level of NICU quality of developmental care and neurobehavioral performance in very preterm infants. Pediatrics 2012;129:e1129-37.

16. Montirosso R, Casini E, Del Prete A, et al. Neonatal developmental care in infant pain management and internalizing behaviors at 18 months in prematurely born children. Eur J Pain 2016;20:1010-21.

17. Lau KM, Chow SM, Lo SK. Parents' perception of the quality of life of preschool children at risk or having developmental disabilities. Qual Life Res 2006;15:1133-41.

18. Lee CF, Hwang FM, Chen CJ, Chien LY. The interrelationships among parenting stress and quality of life of the caregiver and preschool child with very low birth weight. Fam Community Health 2009;32:228-37.

19. Maurice-Stam H, Oort FJ, Last BF, Brons P, Caron HN, Grootenhuis MA. Longitudinal assessment of health-related quality of life in preschool children with non-CNS cancer after the end of successful treatment. Pediatr Blood Cancer 2008;50.5:1047-1051.

20. Rajmil L, Abad S, Sardon O, et al. Reliability and validity of the Spanish version of the TAPQOL: a health-related quality of life (HRQOL) instrument for 1- to 5-year-old children. Int J Nurs Stud 2011;48:549-56.

21. Montirosso R, Fedeli C, Del Prete A, Calciolari G, Borgatti R; NEOACQUA Study Group. Maternal stress and depressive symptoms associated with quality of developmental care in 25 Italian Neonatal Intensive Care Units: a cross sectional observational study. Int J Nurs Stud 2014;51: 994-1002.

22. Zupancic JA, Richardson DK, Horbar JD, Carpenter JH, Lee SK, Escobar GJ; Vermont Oxford Network SNAP Pilot Project Participants. Revalidation of the Score for Neonatal Acute Physiology in the Vermont Oxford Network. Pediatrics 2007;119:e156-63.

23. Hollingshead AB. Four factor index of social status. 1975; New Haven CT: Yale University.

24. Ireton H. The Child Development Inventory Manual Minneapolis, MN: Behavior Science Systems; 1992.

25. Doig KB, Macias MM, Saylor CF, Craver JR, Ingram PE. The Child Development Inventory: A developmental outcome measure for follow-up of the high-risk infant. J Pediatr 1999;135:358-62.

26. Abidin RR. Parenting Stress Index (PSI). Charlottesville, VA: Pediatric Psychology Press, 1990. 